\title{
Effects of surrounding fluid on motility of hyperactivated bovine sperm
}

\author{
Toru Hyakutake ${ }^{\mathrm{a}, *}$, Kotaro Mori $^{\mathrm{b}}$, Koichi Sato $^{\mathrm{b}}$ \\ ${ }^{a}$ Faculty of Engineering, Yokohama National University, 79-5, Hodogaya, Yokohama 240-8501, Japan \\ ${ }^{\mathrm{b}}$ Graduate School of Engineering, Yokohama National University, 79-5, Hodogaya, Yokohama 240-8501, Japan
}

\section{A R T I C L E I N F O}

Article history:

Accepted 5 February 2018

\section{Keywords:}

Bovine sperm

Hyperactivation

Non-Newtonian fluid

Mobility

\begin{abstract}
A B S T R A C T
Mammalian spermatozoa in organisms with internal fertilization are required to swim in the cervical and oviductal mucus, whose rheological properties differ substantially from those of water. Moreover, on the way to the oviduct, a change in sperm motility called hyperactivation may occur. In the present study, we focused on the motion characteristics of hyperactivated bovine sperm and investigated the effect of the surrounding fluid on motility. We prepared two kinds of polyacrylamide with high-viscosity nonNewtonian fluid properties, similar to the actual cervical and oviductal mucus. Using semen from Japanese cattle, we evaluated curvilinear velocity (VCL), straight-line velocity (VSL), and average path velocity (VAP). Additionally, we estimated linearity (LIN), straightness (STR), and wobble (WOB) as sperm motility parameters for several surrounding fluids. We successfully induced hyperactivation of bovine sperm in high-viscosity non-Newtonian fluid. Hyperactivation resulted in an increase in VCL and a decrease in VSL. In the high-viscosity non-Newtonian fluid, the hyperactivated sperm moved in a zigzag pattern with regularity, different from the movement observed in a diluted solution. The increase in WOB in the non-Newtonian fluid suggests that hyperactivated sperm efficiently progress along the groove that exists on the oviductal mucus wall. These results improve our understanding of the motility of bovine sperm when they undergo hyperactivation in the actual cervical and oviductal mucus.
\end{abstract}

(c) 2018 Elsevier Ltd. All rights reserved.

\section{Introduction}

Sperm swimming toward an egg is essential for successful fertilization. The spermatozoa of mammals, including human, bovine, and porcine spermatozoa, generally participate in internal fertilization; therefore, sperm need to swim toward an egg within reproductive organs. The spermatozoa ejaculated in the vagina move forward in the oviduct via the cervical canal and uterus. To reach eggs in the oviduct, mammalian sperm progress by the active motion of the slender flagellum. The considerably long course required for a spermatozoon to meet an egg in female reproductive organs has not been sufficiently clarified. The cervical and oviductal mucus through which sperm travel are composed of various fluids, including macromolecules and gels (Menezo and Guerin, 1997; Lai et al., 2009), whose viscosities are quite high compared to that of water. The mucus also has non-Newtonian fluid properties (Lai et al., 2007). Therefore, to understand the fertilization process, it is critical to examine sperm motility in realistic fluid conditions (see Table 1).

\footnotetext{
* Corresponding author.

E-mail address: hyaku@ynu.ac.jp (T. Hyakutake).
}

On the way to the oviduct, sperm may experience a change in motility, called hyperactivation, mediated by a signal transduction mechanism dependent on the presence of calcium ions. The motility of hyperactivated sperm is characterized by a large flagellar bend amplitude and asymmetrical beating. As a result, hyperactivated sperm move nonprogressively in small circular or figureeight paths, and the straightness of the motion decreases substantially (Yanagimachi, 1970; Katz et al., 1989). Hyperactivation is biologically convenient for sperm penetration through the zona pellucida (Boatman and Robbins, 1991; Yanagimachi, 1994). However, observations of hyperactivated sperm are mostly limited to diluted solutions, and the influence of the actual fluid environment on the motility of hyperactivated sperm is unclear. In the present study, we focused on the effect of the rheological properties of the oviductal mucus on hyperactivated bovine sperm motility.

Several studies have investigated the properties of the cervical and oviductal mucus. Wolf et al. (1977) characterized the mucus in the uterine tube as a viscoelastic fluid containing gelatinous material and macromolecules. Lai et al. (2007) measured the viscosity of fresh human cervical mucus samples as a function of the shear rate and showed that the viscosity of mucus was two orders of magnitude greater than that of water. Their results indicated that the mucus was a non-Newtonian fluid. Some studies 
Table 1

Relationship between shear rate and viscosity for PAA-L and PAA-H.

\begin{tabular}{lll}
\hline Shear rate $\left[\mathrm{s}^{-1}\right]$ & PAA-L $[$ Pa s] & PAA-H $[$ Pa s] \\
\hline 1 & 0.3170 & 1.0500 \\
10 & 0.1470 & 0.2680 \\
100 & 0.0717 & 0.0674 \\
\hline
\end{tabular}

have focused on the effects of the surrounding environment (i.e., the fluid characteristics of the oviduct) on sperm motility, including the effects of fluid viscosity (Hyakutake et al., 2015a, 2017; Katz et al., 1978; Kirkman-Brown et al., 2011; Smith et al., 2009), viscoelastic properties (Tung et al., 2015), and non-Newtonian properties (Hyakutake et al., 2015b). However, the actual viscosity of the mammalian oviductal mucus is much greater than that of fluids in these in vitro experiments. The hyperactivation-inducing signal transduction mechanism elicited by the presence of calcium ions in the medium has been analyzed extensively from a biochemical point of view (Darszon et al., 2001; Harayama et al., 2012; Ignotz and Suarez, 2005; Marquez and Suarez, 2004, 2007; Okamura et al., 1985). Several studies have characterized the motility of hyperactivated sperm. Kaneko et al. (2007) conducted digital image analysis of the flagellar beat of non-hyperactivated and hyperactivated Suncus spermatozoa. Ishijima et al. (2002) reported that the beating frequency of golden hamster spermatozoa decreased in response to hyperactivation. Additionally, Ishijima et al. (2011) reported that lateral force was effective in allowing hyperactivated monkey sperm to penetrate the zona pellucida. A few studies have examined the effect of the surrounding fluid on hyperactivated sperm motility. For example, Suarez et al. (1991) reported that the paths of hyperactivated hamster sperm were straighter in Ficoll solution than in a diluted solution. Additionally, in high-viscosity and viscoelastic solutions, the progressive movement of hyperactivated mouse sperm also increases (Suarez et al., 1992). However, the relationship between the motility of hyperactivated sperm in other mammalian taxa and surrounding fluids is unclear.

In the present study, we focused on the motion characteristics of hyperactivated bovine sperm and investigated how the rheological properties of the oviductal mucus affected motility. The experimental results clarified the mechanics underlying bovine hyperactivated sperm motility under actual environmental conditions, i.e., in a high-viscosity non-Newtonian fluid. Furthermore, the results provide valuable information for improving the success of artificial insemination in animal husbandry and for the development of microfluidic devices to treat infertility (Chen et al., 2011; Cho et al., 2003; Hyakutake et al., 2009; Knowlton et al., 2015; Matsuura et al., 2012; Rappa et al., 2016; Sano et al., 2010; Schuster et al., 2003; Seo, 2007).

\section{Materials and methods}

Sperm motion was observed using an optical microscope (Olympus IX71, Tokyo, Japan) and images were obtained using a CCD camera (K-II; Kato Koken, Isehara, Japan). The study used Japanese cattle semen (Suzukane, Animal Genetics Japan Co., Ltd., Suzuka, Japan), which was cryopreserved in a liquid nitrogen tank. Semen was thawed and supplemented with bovine in vitro fertilization medium (IVF100; Research Institute for the Functional Peptides Co., Ltd., Higashine, Japan). To facilitate observations, bovine semen was separated into sperm and seminal plasma using a centrifugal separator for $10 \mathrm{~min}$, twice. IVF100 was added to the separated sperm, which was then cultured for $3 \mathrm{~h}$. As a result, extreme flagellar bending in the proximal midpiece of sperm was observed from video footage, indicating that hyperactivation of bovine sperm was successfully induced in vitro. After they were cultured, sperm were added to polyacrylamide (PAA) solutions with various rheological properties. The suspension was heated in a water bath at $38.5^{\circ} \mathrm{C}$, and the temperature of the suspension was maintained using a thermoplate during the observation period. The suspension was placed on a glass slide and covered with a coverslip.

A rheometer (ARES-G2; TA Instruments, New Castle, DE, USA) was used to measure the viscosity of the PAA solutions at 38.5 ${ }^{\circ} \mathrm{C}$. Two PAA solutions were used, PAA-L and PAA-H (Wako Pure Chemical Industries, Ltd., Osaka, Japan), which had molecular weights of 5000 and $18,000 \mathrm{kDa}$, respectively. Fig. 1 shows the relationship between shear rate and viscosity for PAA-L, PAA-H, and typical cervical mucus. The viscosity of the diluted solution was similar to that of water and was nearly constant as a function of the shear rate, indicating that it was a Newtonian fluid. The viscosity of the PAA solution was much greater than that of the diluted solution. Additionally, it decreased with an increase in the shear rate. Therefore, the PAA-L and PAA-H solutions were non-Newtonian fluids. The viscosities of PAA-L and PAA-H when the shear rate was $1.0 \mathrm{~s}^{-1}$ were approximately four and fifteen times those when the shear rate was $100 \mathrm{~s}^{-1}$, respectively.

Sperm motion was observed under a microscope and images were obtained at $100 \mathrm{fps}$ using a high-speed camera. For image analysis, a particle-tracking velocimetry technique was applied using DIPP-Motion Pro fluid analysis software (Ditect Co., Ltd., Tokyo, Japan). The trajectory of sperm was obtained by marking the sperm head in the images. Based on the trajectory, three estimates of velocity were obtained using MATLAB (MathWorks, Natick, MA, USA). First, curvilinear velocity (VCL) was calculated by averaging the velocities determined by the change in sperm position for each pair of successive images. Second, straight-line velocity (VSL) was calculated using the distance between sperm position in the first and last frames. Third, average path velocity (VAP) was estimated as the average velocity of sperm cells over a smoothed cell path. In the present experiment, VAP was defined as the velocity over the average path of the sperm calculated as a five-point running average. Additionally, linearity $(\mathrm{LIN}=\mathrm{VSL} / \mathrm{VCL})$, straightness $(\mathrm{STR}=\mathrm{VSL} / \mathrm{VAP})$, and wobble $(\mathrm{WOB}=\mathrm{VAP} / \mathrm{VCL})$ of non-

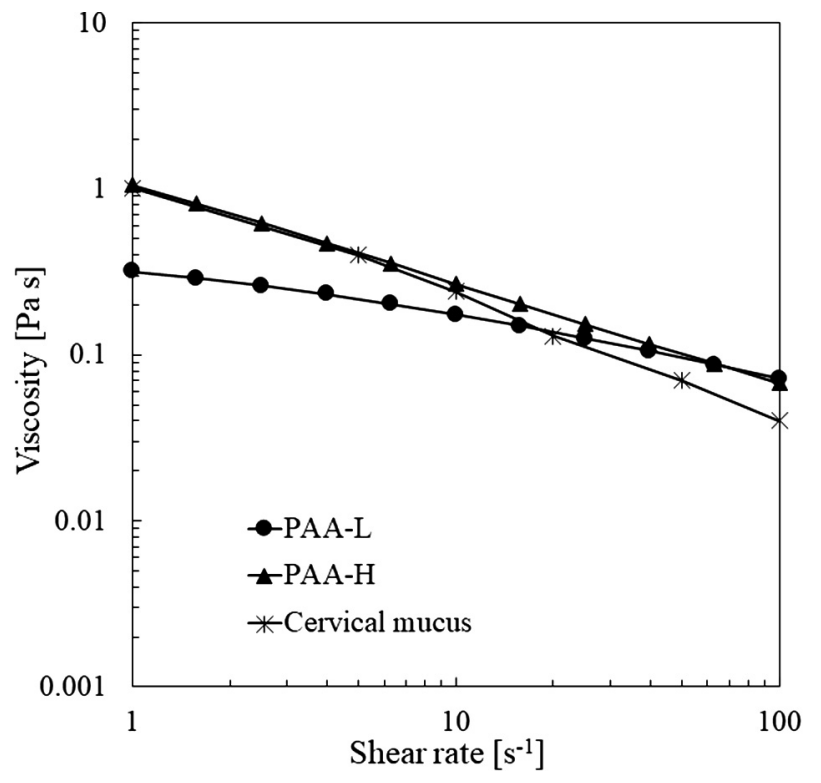

Fig. 1. Relationship between shear rate and viscosity of PAA-L, PAA-H, and human cervical mucus (Lai et al., 2007). PAA-L: filled circles; PAA-H: filled triangles; cervical mucus: asterisks. 
hyperactivated and hyperactivated sperm were compared for various surrounding fluids.

\section{Results}

First, we investigated sperm motility in the absence of polyacrylamide. Fig. 2(a) and (b) shows the typical trajectories of nonhyperactivated and hyperactivated sperm. In the diluted solution, non-hyperactivated sperm usually moved forward in a zig-zag pattern. The motion characteristics of hyperactivated sperm were markedly different from those of non-hyperactivated sperm. The straightness of sperm motion disappeared completely following hyperactivation; instead, these sperm swam in a figure-eight configuration.

Next, we evaluated sperm motion with respect to the properties of the surrounding fluid. Fig. 2(c) and (d) shows the typical trajectories of the non-hyperactivated sperm for low-molecular weight PAA-L and high-molecular weight PAA-H. Motility of nonhyperactivated sperm was lower in the high-viscosity nonNewtonian fluid than in the low-viscosity fluid. Wobble of these sperm decreased and straightness increased in the high-viscosity fluid.

Fig. 2(e) and (f) shows the typical trajectories of hyperactivated sperm in high-viscosity non-Newtonian fluid. Under these conditions, a regular wobble and slight straightness were observed, even though the latter was less evident than that of non-hyperactivated sperm in the high-viscosity fluid.

To investigate the effect of surrounding fluid properties on nonhyperactivated and hyperactivated sperm motility in more detail, we performed quantitative comparisons of motility. Fig. 3 summarizes VCL, VSL, and VAP for three fluid types surrounding the sperm. These values were obtained from approximately 10 sperms that exhibited the highest motility in the same semen sample. As the viscosity of the fluid increased, VSL, VCL, and VAP declined. Hyperactivation significantly increased VCL, especially in the diluted solution, whereby VCL was fivefold higher than that of non-hyperactivated sperm. Hyperactivation also led to a decrease in VSL, especially in PAA-H. In the diluted solution, VAP was higher for hyperactivated than non-hyperactivated sperm, but in the high-viscosity non-Newtonian fluid, VAP was hardly influenced by hyperactivation.

Fig. 4 summarizes the comparative analysis of other parameters related to sperm motility, i.e., LIN, STR, and WOB, while Table 2 lists the corresponding correlation coefficients. Hyperactivation was associated with a significant decline in LIN for every solution. For instance, in the diluted solution, LIN was more than $80 \%$ lower for hyperactivated sperm than non-hyperactivated sperm. Addition of the PAA solution resulted in a slight increase in LIN. STR of non-hyperactivated sperm was nearly 1.0, especially in the case of PAA; however, it decreased by about half in hyperactivated sperm. WOB of non-hyperactivated sperm decreased slightly as the non-Newtonian property increased. In contrast, WOB of hyperactivated sperm increased gradually as the non-Newtonian property increased.

\section{Discussion}

Many rigorous studies have examined the motion characteristics of sperm. However, studies of the effect of the surrounding fluid on sperm motility are limited. In particular, few studies have examined bovine sperm motility in high-viscosity non-Newtonian fluid. The effect of fluid properties of the cervical and oviductal mucus on the motility of hyperactivated bovine sperm remains unclear. In this study, we successfully induced the hyperactivation of bovine sperm in high-viscosity non-Newtonian fluid, which is similar to cervical and oviductal mucus, and examined motility in several fluid types. The results of this study improve our understanding of bovine sperm motility after hyperactivation in the cervical and oviductal mucus. Currently, good motile sperm is identified based on motility in a diluted solution. However, it remains unclear whether such sperm is actually suitable for fertilization. The present experimental data suggest that hyperactivated sperms with high WOB reach the egg more quickly in a high viscosity non-Newtonian fluid. Therefore, these sperms are likely to be more suitable for fertilization. This finding may contribute to effective sorting of bovine sperm for artificial fertilization in the context of animal husbandry.

Non-hyperactivated sperm moved in a zig-zag pattern in the diluted solution and continued to move in a forward direction. As the viscosity of the fluid surrounding the sperm increased, the force acting on the sperm increased. As a result, sperm motility parameters (VCL, VSL, and VAP) were lower for the two PAA solutions than for the diluted solution (Fig. 3). LIN, STR, and WOB were slightly higher in the PAA solutions than in the diluted solution (Fig. 4). In a previous study (Hyakutake, 2015), an increase in the viscosity of a Newtonian fluid led to a decrease not only in sperm motility, but also in linearity, whereas a high-viscosity nonNewtonian fluid decreased sperm motility and increased linearity. In this study, similar results were obtained. The thickness of the flagellum is nonuniform, and it gradually tapers from the midpiece to the endpiece. In non-Newtonian fluids, the thickness of the flagellum has a substantial effect on the local curvature of the flagellum. Consequently, a change in flagellar shape leads to an increase in the linearity of sperm. These results suggest that straightness is maintained, despite the high fluid viscosity, because actual cervical and oviductal mucus have non-Newtonian properties, and this motion is effective for fertilization, i.e., it promotes rapid movement to the egg.

The motility of hyperactivated sperm is characterized by a large flagellar bend amplitude and asymmetrical beating. In the diluted solution, hyperactivated sperm moved nonprogressively and straightness of the sperm motion disappeared (Fig. 2(b)). This nonprogressive movement is inconvenient for fertilization because it decreases the probability that the sperm reach the egg. These results indicated that high-viscosity non-Newtonian properties, similar to the actual fluid properties encountered in the cervix and oviducts (Hyakutake et al., 2015), promote straightness. Accordingly, our experimental observations may be applicable to movement in the cervical and oviductal mucus. Viscosity varies according to the distance from the egg, and increases in the proximity of the egg. We speculate that sperm are adapted to maintain straightness even in high-viscosity fluids by induction of hyperactivation.

In the high-viscosity non-Newtonian fluid, the hyperactivated sperm regularly moved in a zig-zag motion, differing from sperm behavior in the diluted solution (Fig. 2(d) and (f)). LIN and STR were low, and WOB gradually increased as the non-Newtonian properties increased (Fig. 4). These observations can be explained by the cervical wall conditions. In the in vitro experiment, LIN and STR were small because sperm move in a curved pattern. At the micro scale, the mammalian oviduct has a grooved wall, as reported in humans (Sokol, 2011), bovines (Yániz et al., 2000), and mice (Burton et al., 2015). Sperm are expected to swim near the bottom of the groove. Consequently, the curvature may disappear in the groove, and WOB, LIN, and STR may also increase in actual conditions. These results suggest that bovine sperm move effectively for fertilization by increasing straightness in the cervical and oviductal mucus, even after hyperactivation.

Suarez et al. (1991) investigated the effect of hyperactivation on mouse sperm motility, using PAA with molecular weight of $6000 \mathrm{kDa}$, which is close to that of PAA-L in this study. Similar 


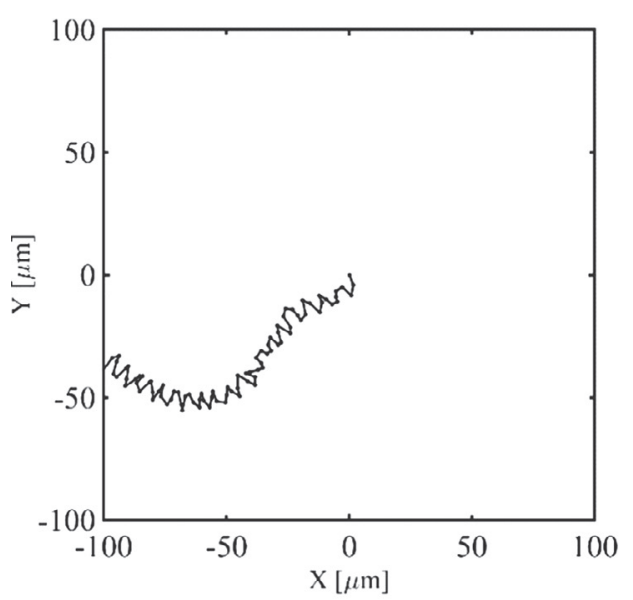

(a) Diluted solution (Non-HA)

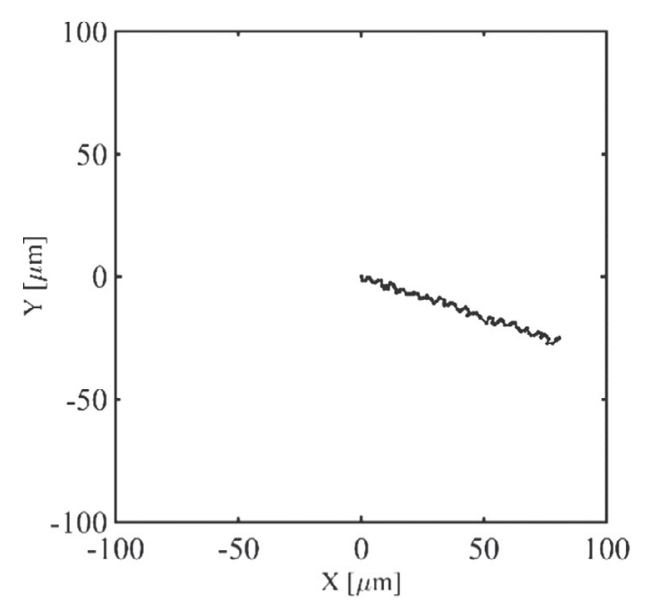

(c) PAA-L (Non-HA)

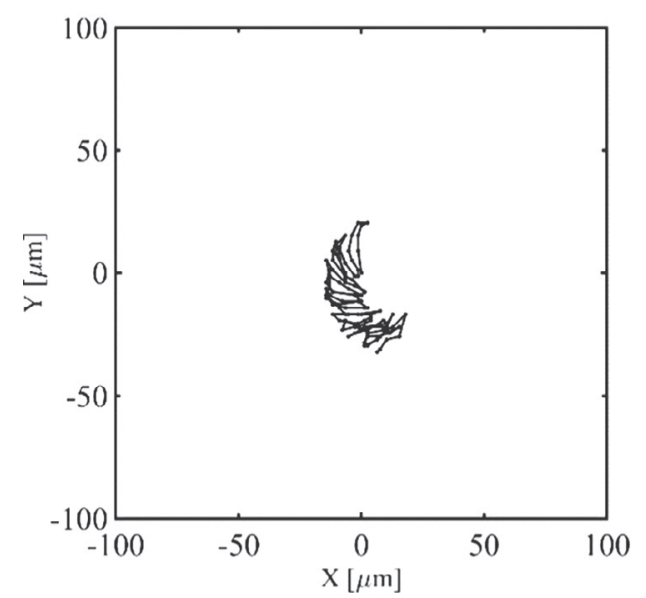

(e) PAA-L (HA)

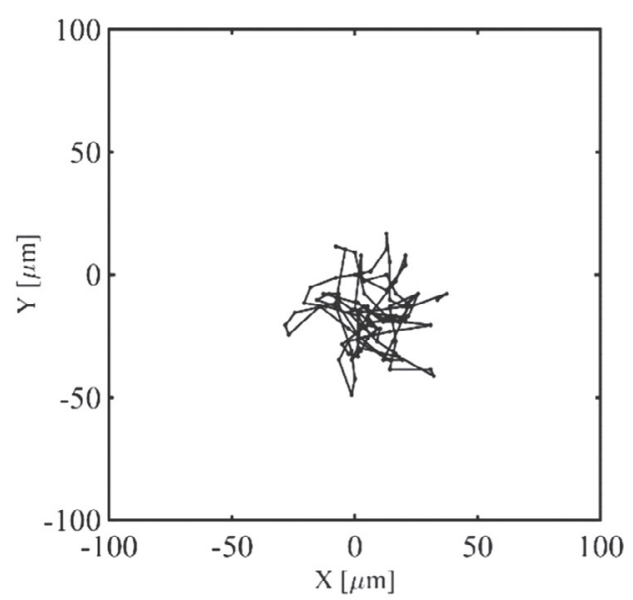

(b) Diluted solution (HA)

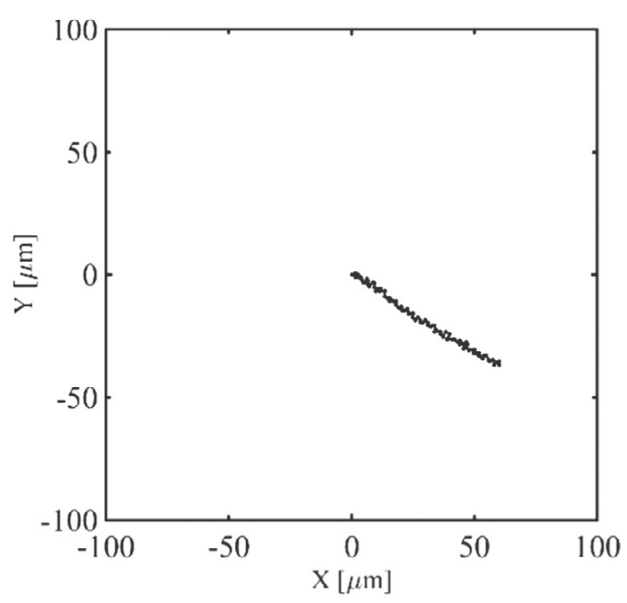

(d) PAA-H (Non-HA)

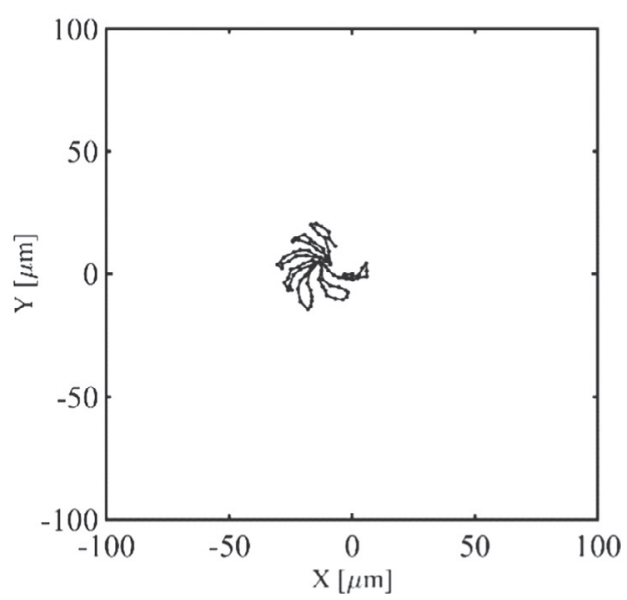

(f) PAA-H (HA)

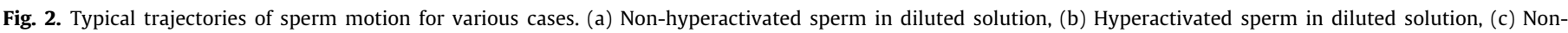
hyperactivated sperm in PAA-L, (d) Non-hyperactivated sperm in PAA-H, (e), Hyperactivated sperm in PAA-L (f) Hyperactivated sperm in PAA-H.

to the present results for bovine sperm, VCL and VAP increased after hyperactivation. However, VSL of mouse sperm in the diluted solution decreased following hyperactivation and increased in the
PAA solution. Our results for bovine sperm indicated that VSL decreased in the PAA solution as well as in the diluted solution after hyperactivation. The most important difference between 


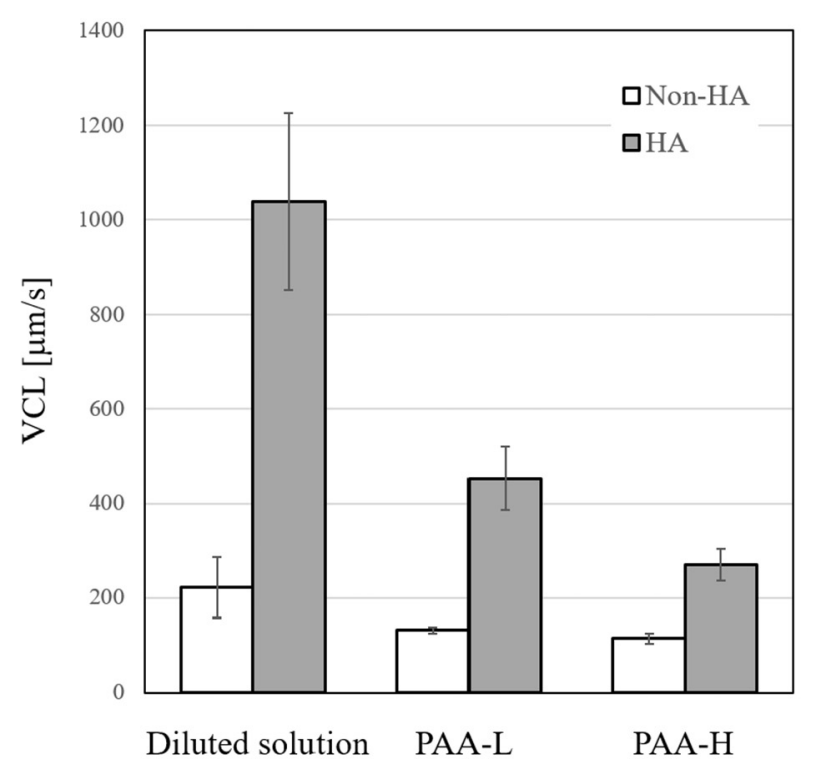

(a)

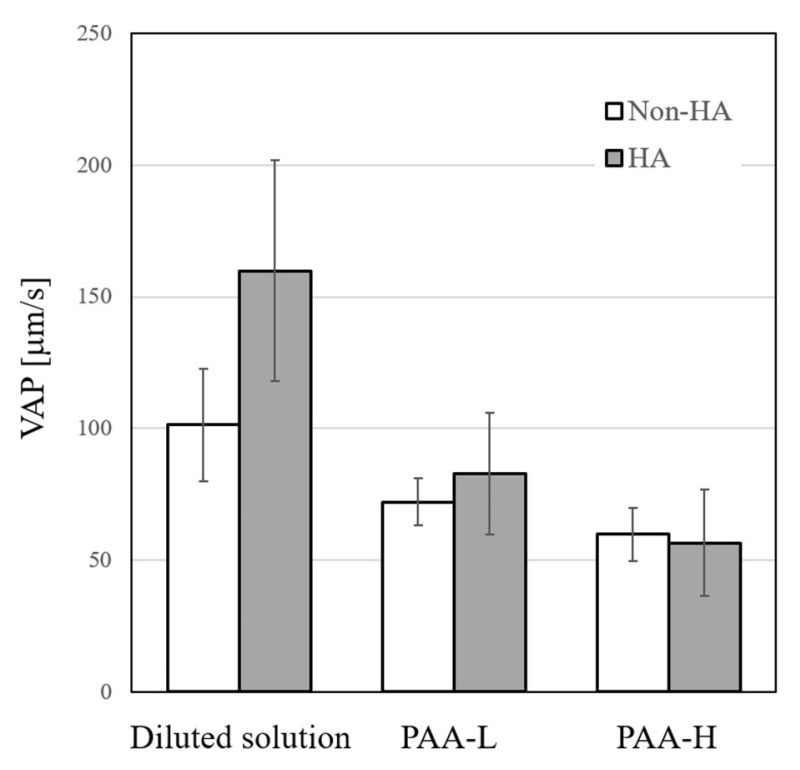

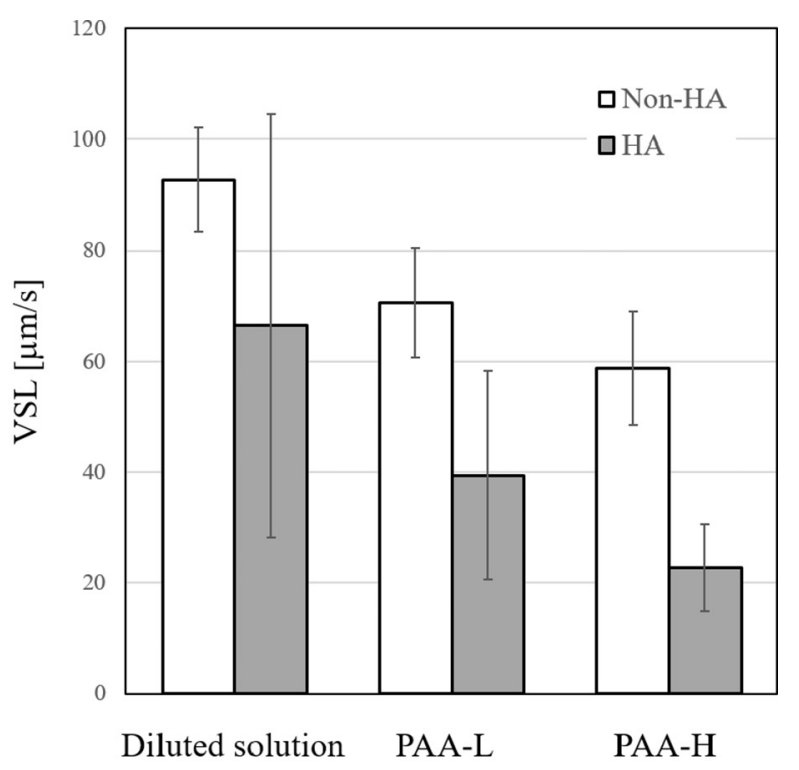

(b)

(c)

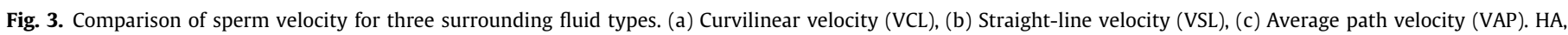
hyperactivated sperm; Non-HA, non-hyperactivated sperm.

the experimental conditions used in the present study and in the study by Suarez et al. (1991) is culture time. This was $3 \mathrm{~h}$ in the present study and $1 \mathrm{~h}$ in that by Suarez et al. Kinukawa et al. (2003) reported that an increase in culture time caused a decrease in linearity. Therefore, we believe that the main contribution to the difference in VSL between the present study and the one by Suarez et al. comes from the use of different culture times. In the actual environment, bovine sperm are expected to swim near the wall, which has microgrooves, leading to an increase in STR. An investigation of the effect of the cervical wall conditions on LIN, STR, and WOB may provide a more detailed understanding of actual sperm motility properties. Moreover, the head shape and flagellar length of bovine sperm differ from those of mouse sperm. It is very interesting that the effects of non-Newtonian properties on the hyperactivated sperm velocity differ among species.

\section{Conclusions}

In the present study, we experimentally investigated the effect of the surrounding fluid on hyperactivated bovine sperm motility. We prepared two polyacrylamide fluids with high-viscosity nonNewtonian fluid properties similar to the surrounding fluid. Based on the sperm trajectory obtained by image analysis, we estimated sperm velocity (VCL, VSL, and VAP) and sperm motility (LIN, STR, 


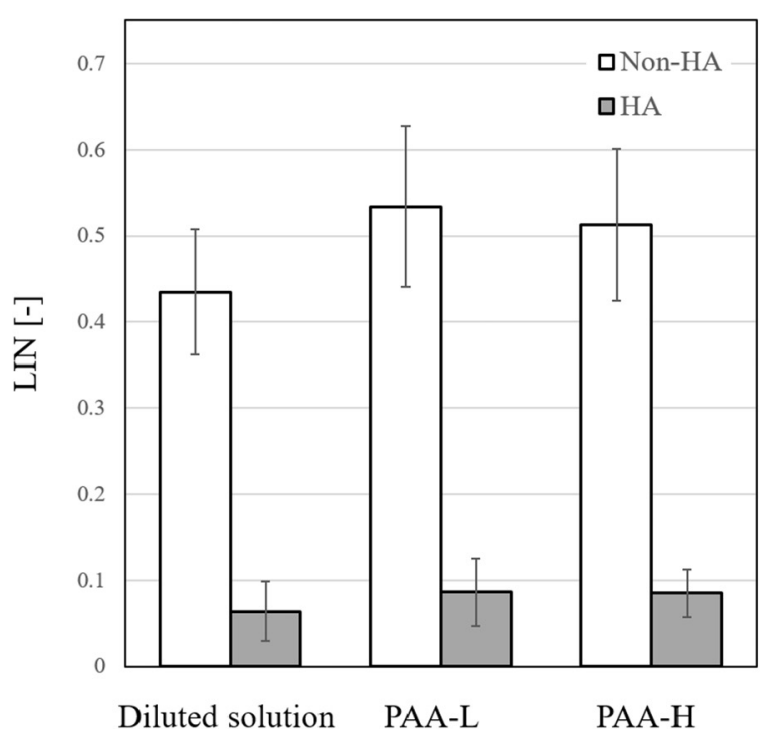

(a)

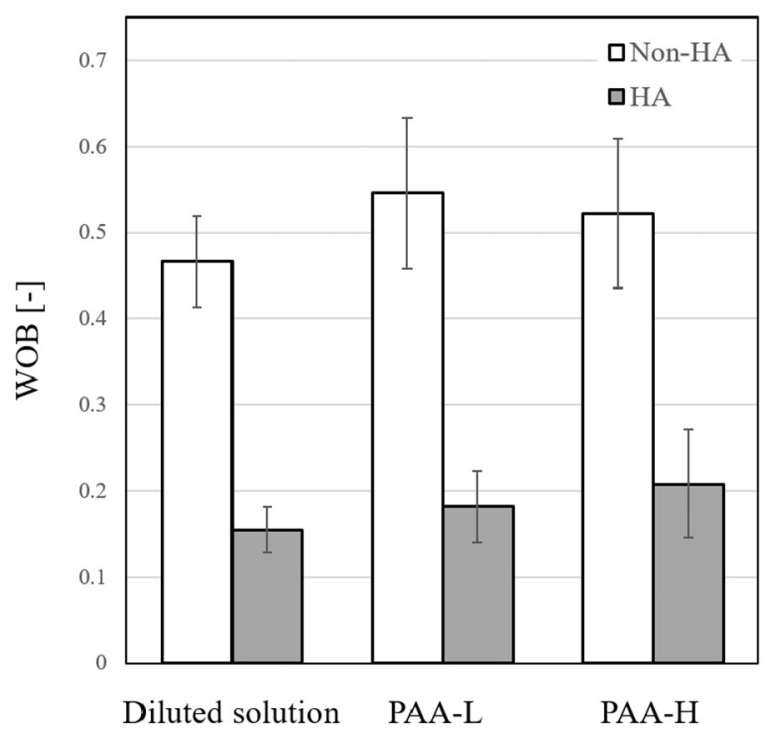

(c)

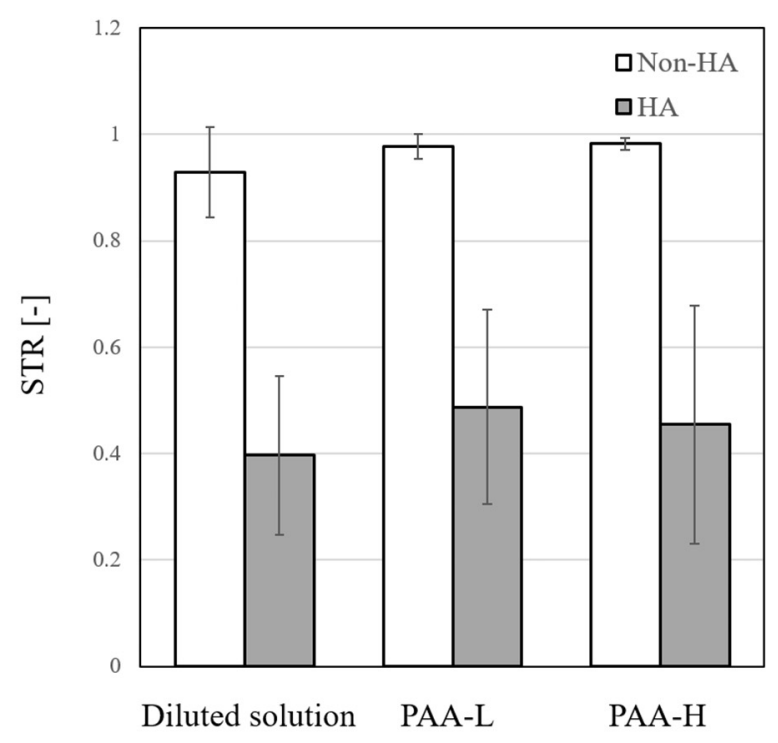

(b) sperm; Non-HA, non-hyperactivated sperm.

Table 2

Correlation coefficients of LIN, STR, and WOB for various cases.

\begin{tabular}{|c|c|c|c|c|c|c|}
\hline & \multicolumn{3}{|l|}{ Non-HA } & \multicolumn{3}{|l|}{$\mathrm{HA}$} \\
\hline & LIN & STR & WOB & LIN & STR & WOB \\
\hline Diluted solution & 0.908 & 0.951 & 0.929 & 0.402 & 0.878 & 0.758 \\
\hline PAA-L & -0.605 & 0.993 & -0.656 & 0.278 & 0.384 & 0.592 \\
\hline PAA-H & 0.216 & 0.998 & 0.242 & 0.247 & -0.063 & 0.559 \\
\hline
\end{tabular}

and WOB). Experimental results indicated that the motion characteristics of bovine sperm varied substantially according to the properties of the surrounding fluid. Hyperactivation led to a significant increase in VCL and a decrease in VSL. In the high-viscosity non-Newtonian fluid, the hyperactivated sperm moved in a zigzag pattern with regularity, differing from the movement observed in the diluted solution. In particular, an increase in WOB in nonNewtonian fluids suggests that actual hyperactivated sperm move efficiently along the groove of the oviductal mucus wall. These results improve our understanding of the motility of hyperactivated bovine sperm under realistic environmental conditions in high-viscosity non-Newtonian fluid. 


\section{Acknowledgement}

This research was supported by a Grant-in-Aid for Challenging Exploratory Research (No. 26560204) from the Japan Society for the Promotion of Science.

\section{Conflict of interest statement}

The authors confirmed that there are no known conflicts of interest associated with this publication and there has been no significant financial support for this work that could have influenced its outcome.

\section{Appendix A. Supplementary material}

Supplementary data associated with this article can be found, in the online version, at https://doi.org/10.1016/j.jbiomech.2018.02. 009.

\section{References}

Boatman, D.E., Robbins, R.T., 1991. Bicarbonate: carbon-dioxide regulation of sperm capacitation, hyperactivated motility, and the acrosome reaction. Biol. Reprod. $44,806-813$.

Burton, J.C., Wang, S., Stewart, C.A., Behringer, R.R., Larina, I.V., 2015. Highresolution three-dimensional in vivo imaging of mouse oviduct using optical coherence tomography. Biomed. Opt. Express 6 (7), 2713-2723.

Chen, Y.-A., Huang, Z.W., Tsai, F.S., Chen, C.Y., Lin, C.M., Wo, A., 2011. Analysis of sperm concentration and motility in a microfluidic device. Microfluid. Nanofluid. 10, 59-67.

Cho, B.S., Schuster, T.G., Zhu, X., Chang, D., Smith, G.D., Takayama, S., 2003. Passively driven integrated microfluidic system for separation of motile sperm. Anal. Chem. 75 (5), 1671-1675.

Darszon, A., Beltràn, C., Felix, R., Nishigaki, T., Treviño, C.L., 2001. Ion transport in sperm signaling. Dev. Biol. 240 (1), 1-14.

Harayama, H., Noda, T., Ishikawa, S., Shidara, O., 2012. Relationship between cyclic AMP-dependent protein tyrosine phosphorylation and extracellular calcium during hyperactivation of boar spermatozoa. Mol. Reprod. Dev. 79 (10), 727 739.

Hyakutake, T., Hashimoto, Y., Yanase, S., Matsuura, K., Naruse, K., 2009. Application of a numerical simulation to improve the separation efficiency of a sperm sorter. Biomed. Microdev. 11 (1), 25-33.

Hyakutake, T., Suzuki, H., Yamamoto, S., 2015a. Effect of viscosity on motion characteristics of bovine sperm. J. Aero Aqua Bio-Mech. 4 (1), 63-70.

Hyakutake, T., Suzuki, H., Yamamoto, S., 2015b. Effect of non-Newtonian fluid properties on bovine sperm motility. J. Biomech. 48, 2941-2947.

Hyakutake, T., Orihara, R., Mezaki, Y., 2017. Experimental study on the effect of a surrounding fluid on bovine sperm motility in three dimensions. J. Biomech. Sci. Eng. 12 (1), 1-9.

Ignotz, G.G., Suarez, S.S., 2005. Calcium/calmodulin and calmodulin kinse II stimulate hyperactivation in demembraneted bovine sperm. Biol. Reprod. 73, 519-526.

Ishijima, S., Baba, S.A., Mohri, H., Suarez, S.S., 2002. Quantitative analysis of flagellar movement in hyperactivated and acrosome-reacted golden hamste spermatozoa. Mol. Reprod. Dev. 61 (3), 376-384.

Ishijima, S., 2011. Dynamics of flagellar force generated by a hyperactivated spermatozoon. Reproduction 142, 409-415.

Kaneko, T., Mori, T., Ishijima, S., 2007. Digital image analysis of the flagellar beat of activated and hyperactivated suncus spermatozoa. Mol. Reprod. Dev. 74 (4), $478-485$.
Katz, D.F., Drobnis, E.Z., Overstreet, J.W., 1989. Factors regulating mammalian sperm migration through the female reproductive tract and oocyte vestments. Gamete Res. 22, 443-469.

Katz, D.F., Mills, R.N., Pritchett, T.R., 1978. The movement of human spermatozoa in cervical mucus. J. Reprod. Fertil. 53, 259-265.

Kinukawa, M., Nagata, M., Aoki, F., 2003. Changes in flagellar bending during the course of hyperactivation in hamster spermatozoa. Reproduction 125, 43-51.

Kirkman-Brown, J.C., Smith, D.J., 2011. Sperm motility: is viscosity fundamental to progress? Molecul. Hum. Reprod. 17 (8), 539-544.

Knowlton, S.M., Sadasivam, M., Tasoglu, S., 2015. Microfluidics for sperm research. Trends Biotechnol. 33 (4), 221-229.

Lai, S.K., O'Hanlon, D.E., Harrold, S., Man, S.T., Wang, Y.Y., Cone, R., Hanes, J., 2007. Rapid transport of large polymeric nanoparticles in fresh undiluted human mucus. Proc. Nat. Acad. Sci. USA 104 (5), 1482-1487.

Lai, S.K., Wang, Y.Y., Wirtz, D., Hanes, J., 2009. Micro- and macrorheology of mucus. Adv. Drug Deliv. Rev. 61, 86-100.

Marquez, B., Suarez, S.S., 2004. Different signaling pathways in bovine sperm regulate capacitation and hyperactivation. Biol. Reprod. 70, 1626-1633.

Marquez, B., Suarez, S.S., 2007. Bovine sperm hyperactivation is promoted by alkaline-stimulated $\mathrm{Ca}^{2+}$ influx. Biol. Reproduct. 76, 660-665.

Matsuura, K., Takenami, M., Kuroda, Y., Hyakutake, T., Yanase, S., Naruse, K., 2012. Screening of sperm velocity by fluid mechanical characteristics of a cyclo-olefin polymer microfluidic sperm-sorting device. Reproduct. Biomed. Onl. 23 (3), 109-115.

Menezo, Y., Guerin, P., 1997. The mammalian oviduct: biochemistry and physiology. Eur. J. Obstet. Gynecol. Reprod. Biol. 73, 99-104.

Okamura, N., Tajima, Y., Soejima, A., Masuda, H., Sugita, Y., 1985. Sodium bicarbonate in seminal plasma stimulates the motility of mammalian spermatozoa through direct activation of adenylate cyclase. J. Biol. Chem. 260, 9699-9705.

Rappa, K.L., Rodriguez, H.F., Hakkarainen, G.C., Anchan, R.M., Mutter, G.L., Asghar, W., 2016. Sperm processing for advanced reproductive technologies: Where are we today? Biotechnol. Adv. 34, 578-587.

Sano, H., Matsuura, K., Naruse, K., Funahashi, H., 2010. Application of a microfluidic sperm sorter to the in-vitro fertilization of porcine oocytes reduced the incidence of polyspermic penetration. Theriogenology 74, 863-870.

Schuster, T.G., Cho, B., Keller, L.M., Takayama, S., Smith, G.D., 2003. Isolation of motile spermatozoa from semen samples using microfluidics. Reproduct. Biomed. Onl. 7 (1), 75-81.

Seo, D.-B., Agca, Y., Feng, Z., Critser, J. 2007. Development of sorting, aligning, and orienting motile sperm using microfluidic device operated by hydrostatic pressure. Microfluid. Nanofluid. 3, 561-570.

Smith, D.J., Gaffiney, E.A., Gadêlha, H., Kapur, N., Kirkman-Brown, J.C., 2009. Bend propagation in the flagella of migrating human sperm, and its modulation by viscosity. Cell Mot. Cytoskelet. 66, 220-236.

Sokol, E., 2011. Glob. Libr. Women's Med. (ISSN: 1756-2228), doi:10.3843/ GLOWM.10001.

Suarez, S.S., Katz, D.F. Owen, D.H., Andrew, J.B., Powell, R.L., 1991. Evidence for the function of hyperactivated motility in sperm. Biol. Reprod. 44 (2), 375-381.

Suarez, S.S., Dai, X., 1992. Hyperactivation enhances mouse sperm capacity for penetrating viscoelastic media. Biol. Reprod. 46 (4), 686-691.

Tung, C., Hu, L., Fiore, A.G., Ardon, F., Hickman, D.G., Gilbert, R.O., Suarez, S.S., Wu, M., 2015. Microgrooves and fluid flows provide preferential passageways for sperm over pathogen Tritrichomonas foetus. Proc. Nat. Acad. Sci. USA 112 (17), 5431-5436.

Wolf, D.P., Blasco, L., Khan, M.A., Litt, M., 1977. Human cervical mucus. I. Rheologic characteristics. Fert. Steril. 28 (1), 41-46.

Yanagimachi, R., 1994. Mammalian fertilization. In: Knobil, E., Neill, J.D. (Eds.), The Physiology of Reproduction. second ed. Raven Press, New York, pp. 189-317.

Yanagimachi, R., 1970. The movement of golden hamster spermatozoa before and after capacitation. J. Reprod. Fertil. 23, 193-196.

Yániz, J.L., Lopez-Gatius, F., Santolaria, P., Mullins, K.J., 2000. Study of the functional anatomy of bovine oviductal mucosa. Anat. Rec. 260 (3), 268-278. 